\title{
A Panel of CA19-9, Ca125, and Ca15-3 as the Enhanced Test for the Differential Diagnosis of the Pancreatic Lesion
}

\author{
Piotr Hogendorf, ${ }^{1}$ Aleksander Skulimowski, ${ }^{1}$ Adam Durczyński, ${ }^{1}$ Anna Kumor, \\ Grażyna Poznańska, ${ }^{3}$ Aleksandra Oleśna, ${ }^{1}$ Joanna Rut, ${ }^{1}$ and Janusz Strzelczyk ${ }^{1}$ \\ ${ }^{1}$ Department of General and Transplant Surgery, Medical University of Lodz, 22 Kopcinskiego St., 90-153 Lodz, Poland \\ ${ }^{2}$ Department of Pulmonology and Allergy, Medical University of Lodz, 22 Kopcinskiego St., 90-153 Lodz, Poland \\ ${ }^{3}$ Department of Anesthesiology and Intensive Care, Medical University of Lodz, 22 Kopcinskiego St., 90-153 Lodz, Poland \\ Correspondence should be addressed to Piotr Hogendorf; phog@wp.pl
}

Received 16 October 2016; Revised 29 January 2017; Accepted 1 February 2017; Published 5 March 2017

Academic Editor: Michael Hawkes

Copyright (C) 2017 Piotr Hogendorf et al. This is an open access article distributed under the Creative Commons Attribution License, which permits unrestricted use, distribution, and reproduction in any medium, provided the original work is properly cited.

\begin{abstract}
Background. Proper diagnosis of pancreatic lesion etiology is a challenging clinical dilemma. Studies suggest that surgery for suspected pancreatic ductal adenocarcinoma (PDAC) reveals a benign lesion in $5 \%$ to $13 \%$ of cases. The aim of our study was to assess whether routinely used biomarkers such as CA19-9, Ca125, Ca15-3, and CEA, when combined, can potentially yield an accurate test predicting pancreatic lesion etiology. Methods. We retrospectively analyzed data of 326 patients who underwent a diagnostic process due to pancreatic lesions of unknown etiology. Results. We found statistically significant differences in mean levels of all biomarkers. In logistic regression model, we applied levels CA19-9, Ca125, and Ca15-3 as variables. Two validation methods were used, namely, random data split into training and validation groups and bootstrapping. Afterward, we built ROC curve using the model that we had created, reaching AUC $=0,801$. With an optimal cut-off point, it achieved specificity of $81,2 \%$ and sensitivity of 63,10\%. Our proposed model has superior diagnostic accuracy to both CA19-9 $(p=0,0194)$ and CA125 ( $p=0,0026)$. Conclusion. We propose a test that is superior to CA19-9 in a differential diagnosis of pancreatic lesion etiology. Although our test fails to reach exceptionally high accuracy, its feasibility and cost-effectiveness make it clinically useful.
\end{abstract}

\section{Introduction}

Pancreatic ductal adenocarcinoma (PDAC) has a relatively low incidence; however, it is associated with a dismal prognosis. It is the fourth cause of cancer-related deaths worldwide, as only $5 \%$ of patients survive up to 5 years after the diagnosis [1]. Notably, mortality from pancreatic cancer has not decreased as it has for other cancers in recent years [2]. The main reasons underlying this situation are the lack of PDAC specific symptoms, its late manifestation, and high aggressiveness. Another problem that clinicians are facing is the lack of a highly effective treatment strategy for PDAC. Nowadays, the surgical treatment is the only curative option for PDAC, yet only $10-20 \%$ of patients with newly diagnosed PDAC will be suitable for pancreatic resection. Unfortunately, $\mathrm{R} 0$ or R1 resection does not modify patients 5 -year survival rate dramatically, as it is not higher than $7-32 \%[3,4]$.
Taking into account these issues, current research focuses on the early detection of PDAC, as not only does it increase the probability of $\mathrm{R} 0$ resection but also it is associated with longer survival of the patients [5]. Another clinical challenge that is widely studied is the proper diagnosis of etiology of the encountered pancreatic lesion. As there is no $100 \%$ specific and sensitive marker of PDAC and radiological signs of PDAC and inflammatory processes affecting pancreas are often misleading, misdiagnosis is relatively frequent. Studies show that surgery reveals benign lesion in a case of $5 \%$ to $13 \%$ of patients initially diagnosed with PDAC [6-11]. This situation has a great impact on patients' later quality of life as well as their further prognosis. In their randomized controlled trial comparing results of Frey procedure and pylorus preserving pancreatoduodenectomy (PD) for chronic pancreatitis, Bachmann et al. [12] reported that in 15-year followup the quality of life was better after Frey procedure in 
terms of the physical status. Moreover, patients treated with Frey procedure had longer mean survival time $(14.5 \pm 0.8$ versus $11.3 \pm 0.8$ years; $p=0.037)$. Previously published trials also showed that organ sparing procedures such as Frey or Beger procedures yield better outcome than more aggressive treatment with PD [13]. Thus, not only should the ideal biomarker of PDAC be detectable on the early stage of carcinogenesis, but also it should be highly accurate as patients with benign lesions greatly benefit from organ sparing surgical techniques. Currently, there are a number of studies reporting the existence of potential biomarkers possessing such properties [14-18]. Nevertheless, as their performance is yet to be validated and they are still commercially unavailable, there is a need to fully assess the usefulness of routinely used cancer biomarkers. While CA19-9 is considered as a standard for PDAC diagnosis, its deficiency is obvious as it is not secreted by around $10 \%$ of the population because of the lack of a functional Lewis enzyme. Moreover, its high levels are present in other diseases, and its diagnostic accuracy falls significantly in jaundiced patients, to name a few drawbacks [19-21]. Nevertheless, a recent meta-analysis showed that CA19-9 has a diagnostic accuracy of $80 \%$ and remains the most useful PDAC biomarker that is routinely measured [22]. There is a growing body of evidence that other biomarkers, such as Ca125 and Ca15-3, can also play an important role in PDAC diagnosis and the prediction of its resectability or survival among patients after surgery or chemotherapy [23-29].

Considering the differential diagnosis of the pancreatic mass, some studies suggest that Ca125 complements CA199. Namely, their simple combination may yield a test that improves accuracy over the routinely used measurement of CA19-9 [19, 30, 31].

Taking into account these findings, our aim was to assess whether routinely used biomarkers, such as CA19-9, Ca125, Ca15-3, and CEA, when combined, can potentially yield an accurate test predicting pancreatic lesion etiology.

\section{Material and Methods}

We retrospectively analyzed the Department's patients registry in order to identify the patients who underwent the diagnostic process due to pancreatic lesions of unknown etiology. From 2009 to 2015, 400 of such patients were admitted to our Department. Out of them, 326 had a panel of CA19-9, Ca125, Ca15-3, and CEA assessed on the day of the hospital admission. This group of patients was considered as eligible for our study (Figure 1). Briefly, a day before surgery, a 4,9 mL blood sample was collected (Serum Z, Sarstedt) and centrifuged for $10 \mathrm{~min}$ at $3000 \mathrm{rpm}$ at $4^{\circ} \mathrm{C}$. Serum was analyzed by the enzyme linked fluorescence assay (ELFA) using a VIDAS PC analyzer (BioMérieux). In order to divide study population into 2 subgroups, namely, patients with PDAC and patients with a benign lesion, we applied the results of pathohistological examination as a mean of the final diagnosis.

As for statistical analysis, we used Mann-Whitney nonparametrical test to compare mean biomarker levels between groups. The same test was used in order to evaluate an association between gender and the level of biomarkers, separately for a group of PDAC patients and nonmalignant patient. In the same manner, Spearman's correlation test was applied to assess the impact of age on the levels of biomarkers.

Diagnostic performance of a single biomarker was measured by building receiver operating characteristic (ROC) curves and choosing the optimal cut-off point afterward. To compare ROC curves area under the curve (AUC), we used a method developed by DeLong et al. [32].

A combined diagnostic test was created after lntransformation of biomarkers' levels, which were used in logistic regression model later on. Other models, such as logistic regression with interaction terms, were also tested, yet it was the simple logistic regression model that yielded the best results (data not shown).

To validate our model, we randomly divided the study population into the training group (228 patients) and the validation group (98 patients). Moreover, a model created for the training group was validated using bootstrapping method (bias-corrected and accelerated method-BC $\mathrm{B}_{a}$ ) [32] ( $n=1000$ bootstrap data sets). In our study, bootstrapping consisted in randomly resampling the data with replacement and repeatedly creating a logistic regression model. Such an approach ensures that obtained coefficients and 95\% confidence intervals are not biased by a number of samples that radically affect data distribution of a certain biomarker.

Finally, we compared the sensitivity and specificity of the resulting model with those of CA19-9, as up to date it is the only standardly measured biomarker of PDAC that has a diagnostic and a prognostic value [33].

The statistical analysis was performed using IBM SPSS Statistics for Windows, version 20.0 (IBM Corp., Armonk, NY) and MedCalc for Windows, version 13.0 (MedCalc Software, Ostend, Belgium).

Ethical approval for this study (Ethical Committee Number $\mathrm{RNN} / 367 / 12 / \mathrm{KB}$ ) was provided. All patients provided written informed consent for the study.

\section{Results}

The basic demographical data concerning study population is shown in Table 1, while the frequency of various benign lesions is presented in Figure 2. Namely, 50 patients had an inflammatory tumor, and 34 had inflammatory cyst diagnosed, while 4 of them had mucinous cystadenoma of the pancreas. Three patients had intraductal papillary mucinous neoplasm and 1 was diagnosed with arteriovenous malformation.

There were no statistically significant differences associated with gender, while patients with PDAC were statistically significantly older than patients with nonmalignant lesions $(p<0,0001)$. To be exact, age $>65$ was associated with the presence of PDAC (HR: 1,94; 95\% CI: 1,14-3,3; $p=0,015$ ). Moreover, age and high levels of Ca125 and CEA and Ca153 moderately correlated with the presence of the unresectable lesion in PDAC group (Spearman's $\rho_{\text {age }}=0,165, p=0,011$; 
TABLE 1

\begin{tabular}{lcc}
\hline & PDAC group & Benign lesions \\
\hline Mean age $( \pm$ SD) & $62 \pm 9$ & $54 \pm 13$ \\
\hline Gender (female (\%)/male (\%)) & $89(38,03 \%) / 145(61,97 \%)$ & $30(32,61 \%) / 62(67,39 \%)$ \\
\hline Resectable (\%)/unresectable $(\%)$ & $66(28,21 \%) / 168(71,79 \%)$ & $53(57,6 \%) / 39(42,4 \%)$ \\
\hline Stage I/II & 66 & 127 \\
Stage III & 41 & \\
Stage IV & & \\
\hline
\end{tabular}

\section{Department's patients registry}

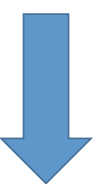

Period from 2009 to 2015 was

analyzed. 750 patients with

pancreatic lesion were identified

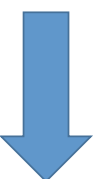

Patients with pancreatic lesion of

uncertain etiology were taken

into account $(n=400)$

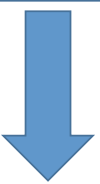

Patients with fully assessed

biomarkers' panel (CA19-9,

Ca125, CEA, and Ca15-3) were

qualified as eligible $(n=326)$

Figure 1

$\rho_{\mathrm{Ca} 15-3}=0,15, p=0,037 ; \rho_{\mathrm{CEA}}=0,195, p=0,006 ; \rho_{\mathrm{Ca} 125}=0,173$, $p=0,024)$.

As for the distribution of biomarker levels, the box plots created with ln-transformed values (Figure 3 ) clearly show that no single biomarker is characterized by the distribution that would entail high performance regarding discriminating a nonmalignant lesion from PDAC. Yet, the differences between mean values of these biomarkers were statistically significant, with CA19-9 and Ca125 being the most significant

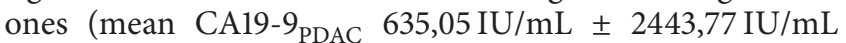
versus mean CA19-9 $9_{\text {benign }} 62,36 \mathrm{IU} / \mathrm{mL} \pm 134,7 \mathrm{IU} / \mathrm{mL}, p<$
0,0001, and mean Ca125 $5_{\mathrm{PDAC}} 45,37 \mathrm{IU} / \mathrm{mL} \pm 89,04 \mathrm{IU} / \mathrm{mL}$ versus mean Ca125 benign $12,74 \mathrm{IU} / \mathrm{mL} \pm 16,65 \mathrm{IU} / \mathrm{mL}, p<$ 0,001) (Table 2).

In accordance with these facts, the ROC curve for CA199 had the highest AUC of 0,736 followed by the ROC curve for Ca125 with AUC of 0,716. Interestingly, when comparing these curves the difference between them was not statistically significant $(p=0,79)$ (Figure 4 and Table 3 ). The AUC of the ROC curves for other biomarkers were not high enough to consider them as independent biomarkers for differentiating pancreatic lesion etiology. 


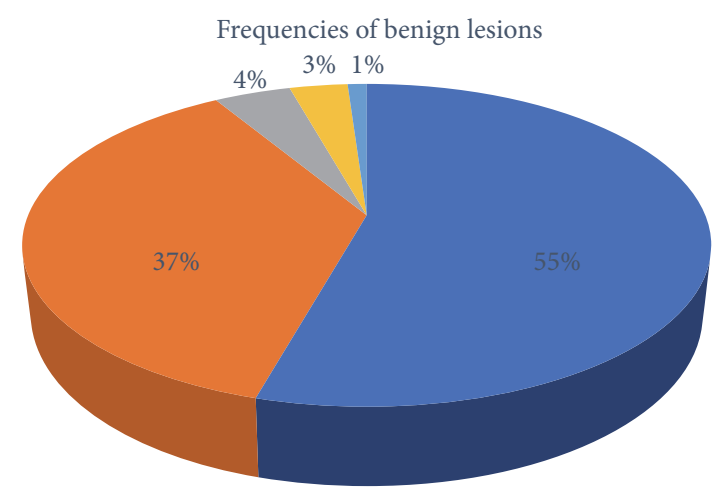

- Inflammatory tumor

- Inflammatory cyst

- Mucinous cystadenoma (MCN)

- Intraductal papillary mucinous neoplasm

- Arteriovenous malformation

Figure 2
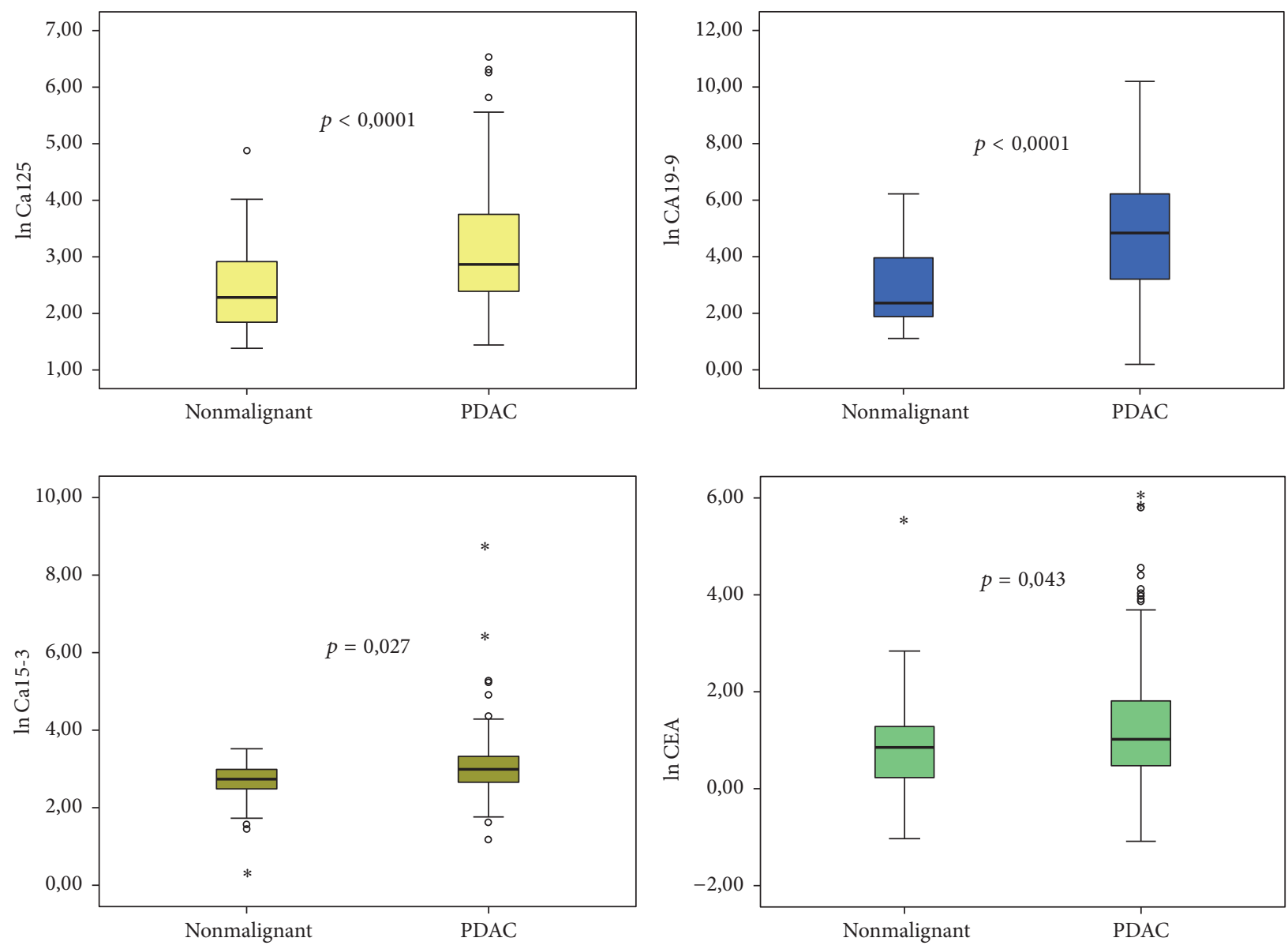

${ }^{\circ}$ Outliner

${ }^{\circ}$ Outliner

${ }^{*}$ Extreme values

FIGURE 3 
TABLE 2

\begin{tabular}{|c|c|c|c|c|}
\hline & & Mean $( \pm \mathrm{SD})[\mathrm{IU} / \mathrm{mL}]$ & $p$ & Area under curve [AUC] $(95 \% \mathrm{CI})$ \\
\hline \multirow{2}{*}{ CA19-9 } & PDAC & $636,05( \pm 2443,77)$ & \multirow{2}{*}{$<0,0001$} & \multirow{2}{*}{$\mathbf{0 , 7 3 6}(0,676-0,795)$} \\
\hline & benign & $62,36( \pm 134,7)$ & & \\
\hline \multirow{2}{*}{ Ca125 } & PDAC & $45,37( \pm 89,05)$ & \multirow{2}{*}{$<0,0001$} & \multirow{2}{*}{$\mathbf{0 , 7 1 7}(0,653-0,782)$} \\
\hline & benign & $12,74( \pm 16,65)$ & & \\
\hline \multirow{2}{*}{ Ca15-3 } & PDAC & $46,03( \pm 301,33)$ & \multirow{2}{*}{0,027} & \multirow{2}{*}{$\mathbf{0 , 5 8 2}(0,515-0,649)$} \\
\hline & benign & $15,77( \pm 7,91)$ & & \\
\hline \multirow{2}{*}{ CEA } & PDAC & $11,95( \pm 44,5)$ & \multirow{2}{*}{0,043} & \multirow{2}{*}{$\mathbf{0 , 5 9 8}(0,527-0,670)$} \\
\hline & benign & $5,44( \pm 25,69)$ & & \\
\hline
\end{tabular}

TABle 3: Pairwise comparison of ROC curves.

\begin{tabular}{lc}
\hline \multicolumn{2}{c}{ Ca125 CA19-9 } \\
\hline Difference between areas & 0,0105 \\
Standard error* & 0,0403 \\
95\% confidence interval & $-0,0683-0,0895$ \\
$Z$ statistics & 0,261 \\
Significance level & $p=0,7942$ \\
\hline
\end{tabular}

${ }^{*}$ DeLong et al. 1988.

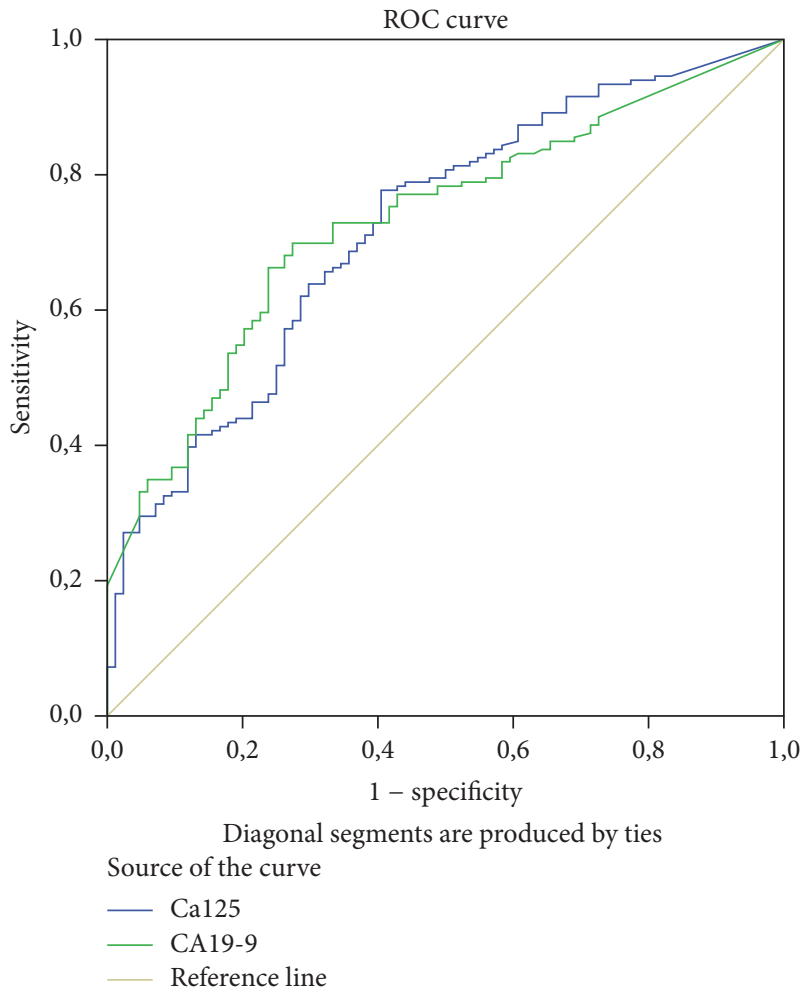

Figure 4

Consequently, using ln-transformed values of biomarkers, we chose logistic regression model as an optimal method for creating a diagnostic test. After splitting the study population randomly into training and validation groups, we applied the chosen model to the training group. We also used the bootstrapping method- $\mathrm{BC}_{a}$ with 1000 data sets. The model summary is shown in Table 4 . The coefficients in our model are as follows: $0,253+1,039 *$ CA19-9 + 1,003*CA125 + $1,048 * C A 15-3$. Afterward, the model was also applied to the validation group and, finally, to the whole study population. As it is shown in Table 5, the AUC of ROC curves built for these groups did not differ significantly $\left(\mathrm{AUC}_{\text {training }}=0,804\right.$; $\mathrm{AUC}_{\text {validation }}=0,791 ; \mathrm{AUC}_{\text {study group }}=0,801$ ).

Judging from these facts, our model is well-fitted not only because of positive cross-validation on both the training and validation group but also because of the fact that the $95 \% \mathrm{CI}$ of coefficients calculated from logistic regression model are closely matching those calculated with the bootstrapping method. Thus, it may be assumed that the calculated model is not biased by the outliers that could highly affect our data.

Using Youden's index, we chose an optimal cut-off point of $\geq 0,5$ for our model, achieving the sensitivity of $81,2 \%$ and specificity of $63,10 \%$. These values outweigh accuracy of CA19-9, which with the clinical cut-off point $(\geq 36 \mathrm{IU} / \mathrm{mL})$ has the sensitivity of $58,97 \%$ and specificity of $79,35 \%$. Similarly, CA125 with optimal cut-off point $\geq 8,5 \mathrm{IU} / \mathrm{mL}$ is also inferior with the sensitivity of $79 \%$ and specificity of $52,17 \%$. We analyzed this data further by comparing ROC curves (Figure 5 and Table 6). Our proposed model has superior diagnostic accuracy to both CA19-9 $(p=0,0194)$ and CA125 $(p=0,0026)$.

Observed differences in the diagnostic accuracy have clinical ramifications. When analyzing PDAC patients' group, $19,66 \%$ of patients have false negative result of the proposed model, while $41,89 \%$ of patients have false negative result of CA19-9. On the other hand, our model yields $38 \%$ of false positive results, while CA19-9 has $19,56 \%$ of false positive results. In terms of unresectable lesions these changes are not crucial, as the palliative management of these tumors, despite etiology, is quite similar. Therefore, we analyzed subgroups of patients resectable lesions, who benefit most from proper diagnosis, since they can be qualified for the adequate surgical treatment. As shown in Table 7, the proposed model has higher true positive rate in the resectable PDAC subgroup $(69,7 \%$ versus $54,54 \%)$ yet it also has higher rate of false positive results in the subgroup of resectable benign lesions $(22,64 \%$ versus $9,43 \%)$

Weighting risk/benefit ratio in this setting is relatively difficult, as the proposed model outweighs the accuracy of CA19-9 in the detection of resectable PDAC, yet it creates 
TABLE 4

\begin{tabular}{|c|c|c|c|c|c|c|c|}
\hline & \multirow{3}{*}{ OR } & \multirow{3}{*}{$p$} & \multicolumn{2}{|c|}{$95 \% \mathrm{CI}$} & \multicolumn{3}{|c|}{ Bootstrap } \\
\hline & & & \multirow{2}{*}{ Lower limit } & \multirow{2}{*}{ Upper limit } & \multirow{2}{*}{$p$} & \multicolumn{2}{|c|}{$95 \% \mathrm{CI}$} \\
\hline & & & & & & Lower limit & Upper limit \\
\hline CA19-9 & 1,003 & 0,002 & 1,001 & 1,005 & 0,004 & 1,002 & 1,007 \\
\hline Ca125 & 1,039 & 0,009 & 1,010 & 1,070 & 0,02 & 1,016 & 1,084 \\
\hline Cal5-3 & 1,003 & 0,015 & 1,009 & 1,087 & 0,006 & 1,018 & 1,099 \\
\hline Constant & 0,253 & 0,001 & N/A & N/A & 0,001 & 0,115 & 0,420 \\
\hline
\end{tabular}

TABLE 5

\begin{tabular}{|c|c|c|c|c|c|}
\hline & \multirow{2}{*}{ Area under curve (AUC) } & \multirow{2}{*}{ SD } & \multirow{2}{*}{$p$} & \multicolumn{2}{|c|}{$95 \% \mathrm{CI}$} \\
\hline & & & & Lower limit & Upper limit \\
\hline Training group & 0,804 & 0,032 & $<0,0001$ & 0,741 & 0,867 \\
\hline Validation group & 0,791 & 0,055 & $<0,0001$ & 0,683 & 0,899 \\
\hline Study group & 0,801 & 0,028 & $<0,0001$ & 0,746 & 0,855 \\
\hline
\end{tabular}

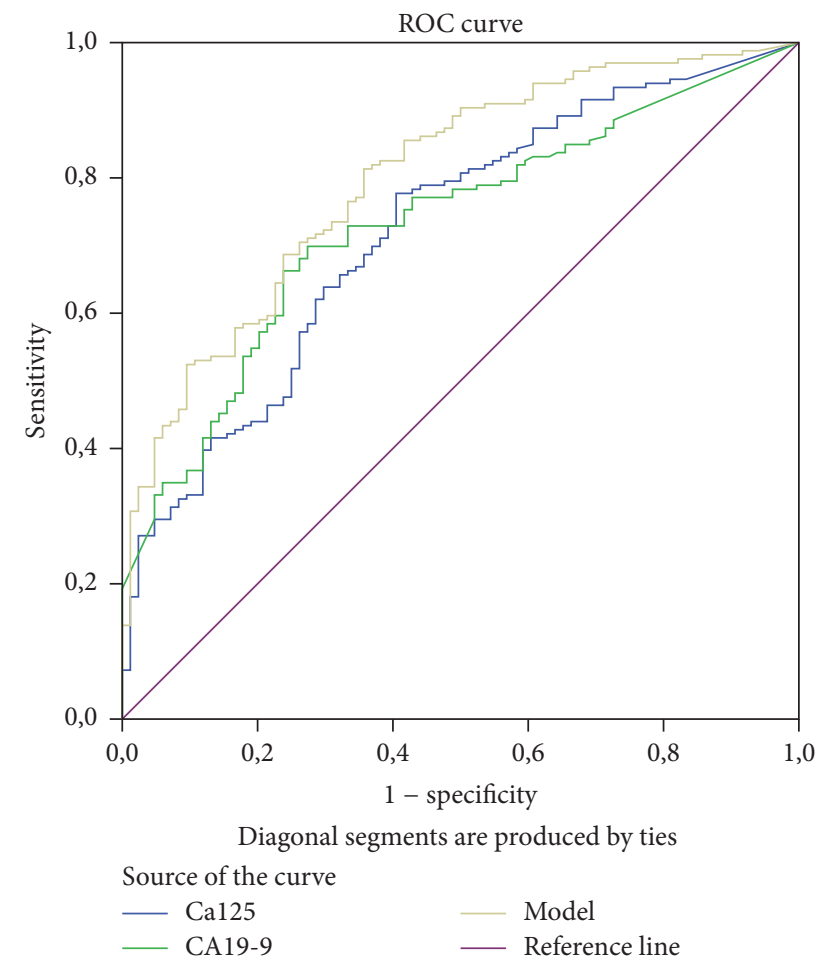

FIGURE 5

a higher risk of qualifying patients with a benign lesion to the PD. However, taking into account the fact that PD is the only curative treatment option for PDAC and it significantly improves the survival it seems that this advantage makes our model superior to the CA19-9 alone. Moreover, it should be also underlined that in some cases of inflammatory tumors Whipple procedure is preferred because of tumor's size or/ and the infiltration of the neighboring tissue. Therefore, in our opinion the improvement of the diagnostic accuracy of the early stage PDAC is a vital asset.

\section{Discussion}

Despite current advances in imaging techniques, the minimally invasive procedures, and biomarker research, proper diagnosis of pancreatic cancer is still challenging. As we still lack highly specific and sensitive biomarker of PDAC, recent development in imaging techniques seems promising. Multidetector flow CT (MDCT) and EUS are accepted as the most effective means of PDAC diagnosis. Yamada et al. reported that MDCT has 90,4\% accuracy in distinguishing PDAC from benign lesion [34]. Similarly, Zhang et al. [35] showed that fusing images obtained by PET/CT and contrastenhanced CT yields 94,3\% accuracy in differentiating benign lesion from the malignant one. As for EUS, its modalities, such as contrast-enhanced EUS or EUS with digital image analysis, have similar accuracy to the techniques mentioned above in terms of lesion etiology differentiation [36-38]. Yet, it should be noted that these techniques are not widespread, and up to date and conventional EUS-FNA has much lower sensitivity and moderately lower specificity [38].

Taking into account the fact that these techniques are mainly used in highly specialized institutions that also have experienced clinicians performing these procedures, it seems that advances in PDAC biomarkers might improve this cancer diagnosis rate in institutions of the lower reference level. As symptoms of PDAC are nonspecific and its differentiation with benign lesion is challenging, the early diagnosis of cancer greatly depends on General Practitioner/family doctor and/or community hospitals capabilities.

As intricate imagining techniques are often unavailable, especially in smaller communities and less developed regions, the proper use of available biomarker tests should be highlighted, as they are cost-effective and easy to perform. It should be also noticed that the incidence of benign lesion found on histopathologic examination after pancreaticoduodenectomy is still relatively high; hence novel tools for differential diagnosis are of uttermost importance.

In our study group, patients with PDAC were statistically significantly older than patients with nonmalignant lesions 
TABLE 6

\begin{tabular}{|c|c|c|c|c|c|}
\hline & \multirow{2}{*}{ Difference between areas } & \multirow{2}{*}{ SE } & \multicolumn{2}{|c|}{$95 \% \mathrm{CI}$} & \multirow[b]{2}{*}{$p$} \\
\hline & & & Lower limit & Upper limit & \\
\hline Model versus CA19-9 & 0,0675 & 0,0289 & 0,0109 & 0,124 & 0,0194 \\
\hline Model versus Ca125 & 0,0780 & 0,0259 & 0,0273 & 0,129 & 0,0026 \\
\hline CA19-9 versus Ca125 & 0,0105 & 0,0403 & $-0,0684$ & 0,0895 & 0,7942 \\
\hline
\end{tabular}

TABLE 7

\begin{tabular}{ccccc}
\hline & Sensitivity & Specificity & Proper diagnosis of resectable PDAC $(n)$ & Proper diagnosis of resectable benign lesion $(n)$ \\
\hline CA19-9 & $58,97 \%$ & $79,35 \%$ & $36 / 66(54,54 \%)$ & $48 / 53(90,56 \%)$ \\
\multirow{2}{*}{ Model } & $81,2 \%$ & $63,1 \%$ & $46 / 66(69,7 \%)$ & $41 / 53(77,36 \%)$ \\
& $\Delta=22,23 \%$ & $\Delta=-16,25 \%$ & $\Delta=15,16 \%$ & $\Delta=-13,2 \%$ \\
\hline
\end{tabular}

$(p<0,0001)$. Moreover, age $>65$ and high levels of Ca125 and CEA and Ca15-3 moderately correlated with the presence of an unresectable lesion in PDAC group. These results seem to be corresponding with the incidence of PDAC which is the highest in the 7 th decade of life $[39,40]$.

In the literature, there is no evidence of age-determined bias on PDAC cancer markers assessment. The majority of studies distinguishing chronic pancreatitis or benign pancreatic lesions from PDAC have almost the same age difference between study groups [41]. However, measurement of PDAC markers in the group of age-adjusted groups would be the most appropriate approach [42].

Currently, CA19-9 is the only recognized biomarker, a "gold standard" for PDAC diagnosis and its monitoring. Yet, it is not highly specific or sensitive, as approximately $10 \%$ of the world population do not secrete this molecule due to lack of Lewis antigen [43]. In terms of false positive results, many benign conditions, as well as other cancers, cause CA19-9 levels to increase [20, 44]. Furthermore, studies show that only around $50 \%$ of PDAC with diameter $<20 \mathrm{~mm}$ have significantly increased CA19-9 levels [45]. Thus, Ca 19-9 levels usefulness in early PDAC diagnosis is questionable. Nevertheless, when differentiating pancreatic lesion of unknown etiology, CA19-9 has reportedly the sensitivity and specificity of $75,36 \%$ and $60,60 \%$, respectively, which makes it a relatively useful tool [46].

Ca125 (also known as MUC16) is a protein that has a wellestablished role in the PDAC development, especially in the later stages; for instance, it promotes cancer cells motility and drug-resistance and reprograms PDAC metabolism [4750]. Its clinical utility is also thoroughly assessed. As already mentioned, Ca125 alone is a relatively accurate biomarker of PDAC and its level has also impact on patients prognosis and it can indicate the resectability of a tumor. Yet, it seems that Ca125 reaches significantly high levels during the late stage of PDAC progression $[23,51,52]$. Thus, its relatively high sensitivity is hampered by low specificity.

Ca15-3 is a derivate of MUC-1, a protein that plays an essential role in PDAC development. Namely, some studies show that MUC1 promotes survival of PDAC cells by inducing hypoxia-inducible factor 1 alpha expression and modulating cells metabolism as well as promoting resistance to chemotherapeutics [53-55]. Moreover, MUC1 regulates expression of a great number of miRNAs. There is evidence that this role of $\mathrm{MUC1}$ is crucial for PDAC progression, as, for instance, MUC1 influences transcription of microRNAs that are associated with the process of metastasis [56]. This data might suggest that assessment of Ca15-3 could prove a useful biomarker for PDAC diagnosis and/or patients monitoring. However, reports published up to date and our data do not confirm that Ca15-3 alone can be considered as a good biomarker of PDAC.

\section{Conclusions}

Currently, we still lack a highly accurate, easy-to-perform, and cost-effective singular biomarker of PDAC, which would enable its early diagnosis in virtually every institution. Therefore, combining the measurement of universally available biomarkers to enhance the accuracy of PDAC diagnosis seems promising. In our study, we propose a test consisting of three biomarkers that is superior to CA19-9 in a differential diagnosis of pancreatic lesion etiology. Although our test fails to reach exceptionally high accuracy, its feasibility and costeffectiveness make it clinically useful. Given these features, it can help in determining pancreatic lesion etiology in almost every clinical setting. It might be hypothesized that its results, combined with the results from conventional imagining techniques, can enhance the rate of proper assessment of pancreatic lesion etiology.

\section{Competing Interests}

The authors declare that they have no competing interests.

\section{Authors' Contributions}

Piotr Hogendorf and Aleksander Skulimowski equally contributed to this work.

\section{References}

[1] R. L. Siegel, K. D. Miller, and A. Jemal, "Cancer statistics, 2015," CA: A Cancer Journal for Clinicians, vol. 65, no. 1, pp. 5-29, 2015. 
[2] A. L. Lucas, M. Malvezzi, G. Carioli et al., "Global trends in pancreatic cancer mortality from 1980 through 2013 and predictions for 2017," Clinical Gastroenterology and Hepatology, vol. 14, no. 10, pp. 1452.e4-1462.e4, 2016.

[3] S. S. Nitecki, M. G. Sarr, T. V. Colby, and J. A. Van Heerden, "Long-term survival after resection for ductal adenocarcinoma of the pancreas: is it really improving?" Annals of Surgery, vol. 221, no. 1, pp. 59-66, 1995.

[4] N. Alexakis, C. Halloran, M. Raraty, P. Ghaneh, R. Sutton, and J. P. Neoptolemos, "Current standards of surgery for pancreatic cancer," British Journal of Surgery, vol. 91, no. 11, pp. 1410-1427, 2004.

[5] B. M. Rau, K. Moritz, S. Schuschan, G. Alsfasser, F. Prall, and E. Klar, "R1 resection in pancreatic cancer has significant impact on long-term outcome in standardized pathology modified for routine use," Surgery, vol. 152, no. 3, supplement 1, pp. S103-S111, 2012.

[6] C. J. Yeo, J. L. Cameron, T. A. Sohn et al., "Six hundred fifty consecutive pancreaticoduodenectomies in the 1990s: pathology, complications, and outcomes," Annals of Surgery, vol. 226, no. 3, pp. 248-260, 1997.

[7] C. M. Schmidt, E. S. Powell, C. T. Yiannoutsos et al., "Pancreaticoduodenectomy: a 20-year experience in 516 patients," Archives of Surgery, vol. 139, no. 7, pp. 718-725, 2004.

[8] C. D. Smith, K. E. Behrns, J. A. Van Heerden, and M. G. Sarr, "Radical pancreatoduodenectomy for misdiagnosed pancreatic mass," British Journal of Surgery, vol. 81, no. 4, pp. 585-589, 1994.

[9] J. L. Cameron, H. A. Pitt, C. J. Yeo, K. D. Lillemoe, H. S. Kaufman, and J. Coleman, "One hundred and forty-five consecutive pancreaticoduodenectomies without mortality," Annals of Surgery, vol. 217, no. 5, pp. 430-438, 1993.

[10] U. Boggi, G. Amorese, F. Vistoli et al., "Laparoscopic pancreaticoduodenectomy: a systematic literature review," Surgical Endoscopy and Other Interventional Techniques, vol. 29, no. 1, pp. 9-23, 2015.

[11] H. J. Asbun, K. Conlon, L. Fernandez-Cruz et al., "When to perform a pancreatoduodenectomy in the absence of positive histology? a consensus statement by the international study group of pancreatic surgery," Surgery, vol. 155, no. 5, pp. 887892, 2014.

[12] K. Bachmann, L. Tomkoetter, A. Kutup et al., "Is the whipple procedure harmful for long-term outcome in treatment of chronic pancreatitis? 15-years follow-up comparing the outcome after pylorus-preserving pancreatoduodenectomy and frey procedure in chronic pancreatitis," Annals of Surgery, vol. 258, no. 5, pp. 815-821, 2013.

[13] D. Hartmann and H. Friess, "Surgical approaches to chronic pancreatitis," Gastroenterology Research and Practice, vol. 2015, Article ID 503109, 6 pages, 2015.

[14] E. Vila-Navarro, M. Vila-Casadesús, L. Moreira et al., "MicroRNAs for detection of pancreatic neoplasia: biomarker discovery by next-generation sequencing and validation in 2 independent cohorts," Annals of Surgery, 2016.

[15] Z. Cao, C. Liu, J. Xu et al., "Plasma microRNA panels to diagnose pancreatic cancer: Results from A Multicenter Study," Oncotarget, vol. 7, no. 27, pp. 41575-41583, 2016.

[16] M. K. Bhasin, K. Ndebele, O. Bucur et al., "Meta-analysis of transcriptome data identifies a novel 5-gene pancreatic adenocarcinoma classifier," Oncotarget, vol. 7, no. 17, pp. 2326323281, 2016
[17] Y. G. Hernandez, "MicroRNA in pancreatic ductal adenocarcinoma and its precursor lesions," World Journal of Gastrointestinal Oncology, vol. 8, no. 1, pp. 18-29, 2016.

[18] K. Honda, M. Kobayashi, T. Okusaka et al., "Plasma biomarker for detection of early stage pancreatic cancer and risk factors for pancreatic malignancy using antibodies for apolipoprotein-AII isoforms," Scientific Reports, vol. 5, Article ID 15921, 2015.

[19] M. Ducreux, A. S. Cuhna, C. Caramella et al., "Cancer of the pancreas: ESMO Clinical practice guidelines for diagnosis, treatment and follow-up," Annals of Oncology, vol. 26, pp. v56v68, 2015.

[20] J. L. Parra, S. Kaplan, and J. S. Barkin, "Elevated CA 19-9 caused by Hashimoto's thyroiditis: review of the benign causes of increased CA 19-9 level," Digestive Diseases and Sciences, vol. 50, no. 4, pp. 694-695, 2005.

[21] H.-R. Kim, C.-H. Lee, Y. W. Kim, S. K. Han, Y.-S. Shim, and J.J. Yim, "Increased CA 19-9 level in patients without malignant disease," Clinical Chemistry and Laboratory Medicine, vol. 47, no. 6, pp. 750-754, 2009.

[22] Z. Huang and F. Liu, "Diagnostic value of serum carbohydrate antigen 19-9 in pancreatic cancer: a meta-analysis," Tumor Biology, vol. 35, no. 8, pp. 7459-7465, 2014.

[23] L. Liu, H.-X. Xu, W.-Q. Wang et al., "Serum CA125 is a novel predictive marker for pancreatic cancer metastasis and correlates with the metastasis-associated burden," Oncotarget, vol. 7, no. 5, pp. 5943-5956, 2016.

[24] L. Liu, J. Xiang, R. Chen et al., "The clinical utility of CA125/MUC16 in pancreatic cancer: a consensus of diagnostic, prognostic and predictive updates by the Chinese Study Group for Pancreatic Cancer (CSPAC)," International Journal of Oncology, vol. 48, no. 3, pp. 900-907, 2016.

[25] T. Chen, M.-G. Zhang, H.-X. Xu, W.-Q. Wang, L. Liu, and X.J. Yu, "Preoperative serum CA125 levels predict the prognosis in hyperbilirubinemia patients with resectable pancreatic ductal adenocarcinoma," Medicine, vol. 94, no. 19, article e751, 2015.

[26] Y. Nagashio, S. Hijioka, N. Mizuno et al., "Combination of cyst fluid CEA and CA 125 is an accurate diagnostic tool for differentiating mucinous cystic neoplasms from intraductal papillary mucinous neoplasms," Pancreatology, vol. 14, no. 6, pp. 503-509, 2014.

[27] E. Dotan, R. K. Alpaugh, K. Ruth et al., "Prognostic significance of MUC-1 in circulating tumor cells in patients with metastatic pancreatic adenocarcinoma," Pancreas, vol. 45, no. 8, pp. 1131$1135,2016$.

[28] D. B. Shultz, J. Pai, W. Chiu et al., "A novel biomarker panel examining response to gemcitabine with or without erlotinib for pancreatic cancer therapy in NCIC Clinical Trials Group PA.3," PLoS ONE, vol. 11, no. 1, Article ID e0147995, 2016.

[29] G. Luo, Z. Xiao, J. Long et al., "CA125 is superior to CA19-9 in predicting the resectability of pancreatic cancer," Journal of Gastrointestinal Surgery, vol. 17, no. 12, pp. 2092-2098, 2013.

[30] A. Chan, I. Prassas, A. Dimitromanolakis et al., "Validation of biomarkers that complement CA19.9 in detecting early pancreatic cancer," Clinical Cancer Research, vol. 20, no. 22, pp. 5787-5795, 2014.

[31] L. Coppin, K. Benomar, F. Corfiotti et al., "CA-125, but not galectin-3, complements CA 19-9 for discriminating ductal adenocarcinoma versus non-malignant pancreatic diseases," Pancreatology, vol. 16, no. 1, pp. 115-120, 2016.

[32] E. R. DeLong, D. M. DeLong, and D. L. Clarke-Pearson, "Comparing the areas under two or more correlated receiver 
operating characteristic curves: a nonparametric approach," Biometrics, vol. 44, no. 3, pp. 837-845, 1988.

[33] B. Efron and R. J. Tibshirani, An introduction to the bootstrap, vol. 57 of Monographs on Statistics and Applied Probability, Chapman and Hall, NY, USA, 1994.

[34] Y. Yamada, H. Mori, S. Matsumoto, H. Kiyosue, Y. Hori, and N. Hongo, "Pancreatic adenocarcinoma versus chronic pancreatitis: differentiation with triple-phase helical CT," Abdominal Imaging, vol. 35, no. 2, pp. 163-171, 2010.

[35] J. Zhang, C.-J. Zuo, N.-Y. Jia et al., "Cross-modality PET/CT and contrast-enhanced CT imaging for pancreatic cancer," World Journal of Gastroenterology, vol. 21, no. 10, pp. 2988-2996, 2015.

[36] M. Zhu, C. Xu, J. Yu et al., "Differentiation of pancreatic cancer and chronic pancreatitis using computer-aided diagnosis of endoscopic ultrasound (EUS) images: a diagnostic test," PLoS ONE, vol. 8, no. 5, Article ID e63820, 2013.

[37] J. Zhu, L. Wang, Y. Chu et al., "A new descriptor for computeraided diagnosis of EUS imaging to distinguish autoimmune pancreatitis from chronic pancreatitis," Gastrointestinal Endoscopy, vol. 82, no. 5, pp. 831-836.e1, 2015.

[38] A. K. Dutta and A. Chacko, "Head mass in chronic pancreatitis: inflammatory or malignant," World Journal of Gastrointestinal Endoscopy, vol. 7, no. 3, pp. 258-264, 2015.

[39] S. F. Altekruse, C. L. Kosary, M. Krapcheco et al., SEER Cancer Statistics Review, National Cancer Institute, 1975-2007.

[40] S. Shore, D. Vimalachandran, M. G. T. Raraty, and P. Ghaneh, "Cancer in the elderly: pancreatic cancer," Surgical Oncology, vol. 13, no. 4, pp. 201-210, 2004.

[41] J. H. Balcom IV, D. W. Rattner, A. L. Warshaw, Y. Chang, and C. Fernandez-Del Castillo, "Ten-year experience with 733 pancreatic resections: changing indications, older patients, and decreasing length of hospitalization," Archives of Surgery, vol. 136, no. 4, pp. 391-398, 2001.

[42] L. F. Hutchins, J. M. Unger, J. J. Crowley, C. A. Coltman Jr., and K. S. Albain, "Underrepresentation of patients 65 years of age or older in cancer- treatment trials," New England Journal of Medicine, vol. 341, no. 27, pp. 2061-2067, 1999.

[43] K. S. Goonetilleke and A. K. Siriwardena, "Systematic review of carbohydrate antigen (CA 19-9) as a biochemical marker in the diagnosis of pancreatic cancer," European Journal of Surgical Oncology, vol. 33, no. 3, pp. 266-270, 2007.

[44] D. Marrelli, S. Caruso, C. Pedrazzani et al., "CA19-9 serum levels in obstructive jaundice: clinical value in benign and malignant conditions," American Journal of Surgery, vol. 198, no. 3, pp. 333$339,2009$.

[45] K. Hanada, A. Okazaki, N. Hirano et al., "Effective screening for early diagnosis of pancreatic cancer," Best Practice and Research: Clinical Gastroenterology, vol. 29, no. 6, pp. 929-939, 2015.

[46] Q. Liao, Y.-P. Zhao, Y.-C. Yang, L.-J. Li, X. Long, and S.M. Han, "Combined detection of serum tumor markers for differential diagnosis of solid lesions located at the pancreatic head," Hepatobiliary and Pancreatic Diseases International, vol. 6, no. 6, pp. 641-645, 2007.

[47] K. Jiang, E. Tan, Z. Sayegh, B. Centeno, M. Malafa, and D. Coppola, "Cancer antigen 125 (CA125, MUC16) protein expression in the diagnosis and progression of pancreatic ductal adenocarcinoma," Applied Immunohistochemistry and Molecular Morphology, 2016.

[48] S. Das, S. Rachagani, M. P. Torres-Gonzalez et al., "Carboxylterminal domain of MUC16 imparts tumorigenic and metastatic functions through nuclear translocation of JAK2 to pancreatic cancer cells," Oncotarget, vol. 6, no. 8, pp. 5772-5787, 2015.

[49] S.-H. Chen, W.-C. Hung, P. Wang, C. Paul, and K. Konstantopoulos, "Mesothelin binding to CA125/MUC16 promotes pancreatic cancer cell motility and invasion via MMP-7 activation," Scientific Reports, vol. 3, article no. 1870, 2013.

[50] S. K. Shukla, V. Gunda, J. Abrego et al., "MUC16-mediated activation of mTOR and c-MYC reprograms pancreatic cancer metabolism," Oncotarget, vol. 6, no. 22, pp. 19118-19131, 2015.

[51] D. Haridas, S. Chakraborty, M. P. Ponnusamy et al., "Pathobiological implications of MUC16 expression in pancreatic cancer," PLoS ONE, vol. 6, no. 10, Article ID e26839, 2011.

[52] K.-I. Okada, M. Kawai, M. Tani et al., "Predicting factors for unresectability in patients with pancreatic ductal adenocarcinoma," Journal of Hepato-Biliary-Pancreatic Sciences, vol. 21, no. 9, pp. 648-653, 2014.

[53] S. Nath, K. Daneshvar, L. D. Roy et al., "MUC1 induces drug resistance in pancreatic cancer cells via upregulation of multidrug resistance genes," Oncogenesis, vol. 2, article no. e51, 2013.

[54] S. Kitamoto, S. Yokoyama, M. Higashi, N. Yamada, S. Takao, and $\mathrm{S}$. Yonezawa, "MUC1 enhances hypoxia-driven angiogenesis through the regulation of multiple proangiogenic factors," Oncogene, vol. 32, no. 39, pp. 4614-4621, 2013.

[55] S. Tréhoux, B. Duchêne, N. Jonckheere, and I. Van Seuningen, "The MUC1 oncomucin regulates pancreatic cancer cell biological properties and chemoresistance. Implication of $\mathrm{p} 42$ 44 MAPK, Akt, Bcl-2 and MMP13 pathways," Biochemical and Biophysical Research Communications, vol. 456, no. 3, pp. 757762, 2015.

[56] A. M. Mohr, J. M. Bailey, M. E. Lewallen et al., "MUC1 regulates expression of multiple microRNAs involved in pancreatic tumor progression, including the miR-200c/141 cluster," PLoS ONE, vol. 8, no. 10, Article ID e73306, 2013. 


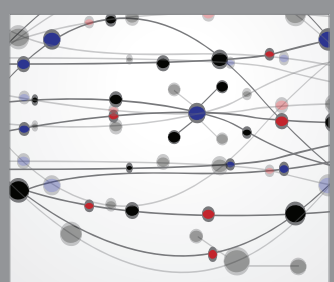

The Scientific World Journal
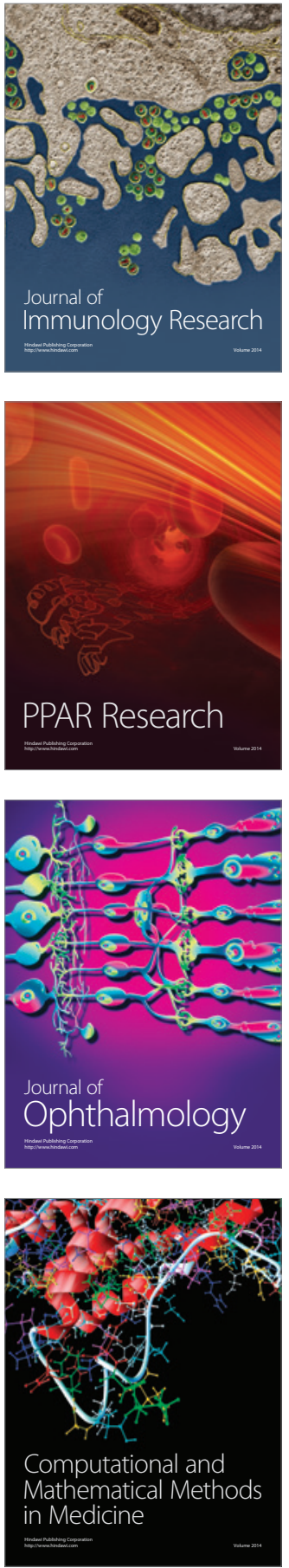

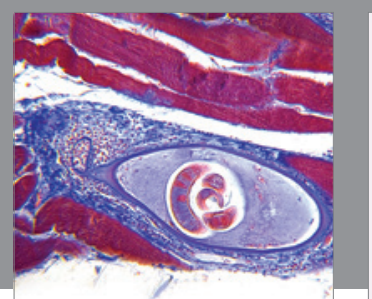

Gastroenterology Research and Practice
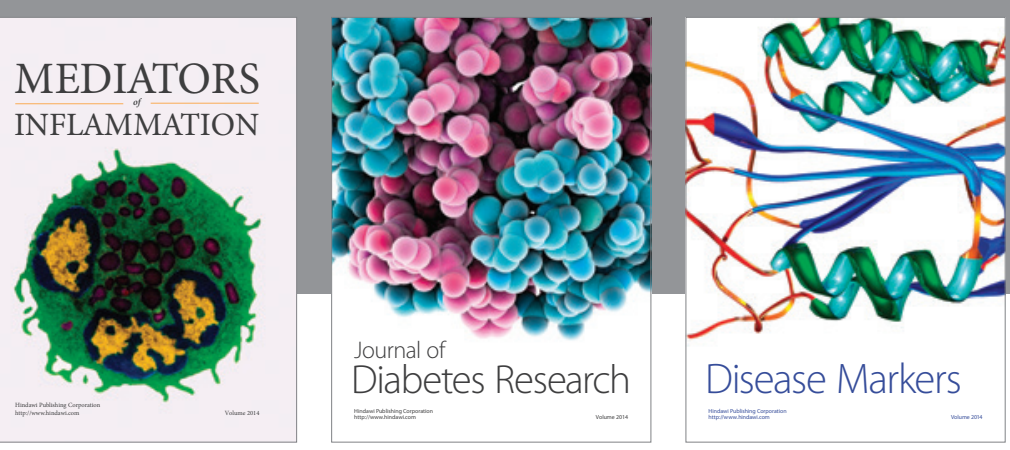

Disease Markers

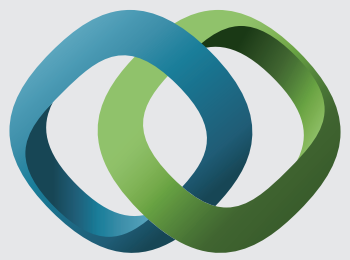

\section{Hindawi}

Submit your manuscripts at

https://www.hindawi.com
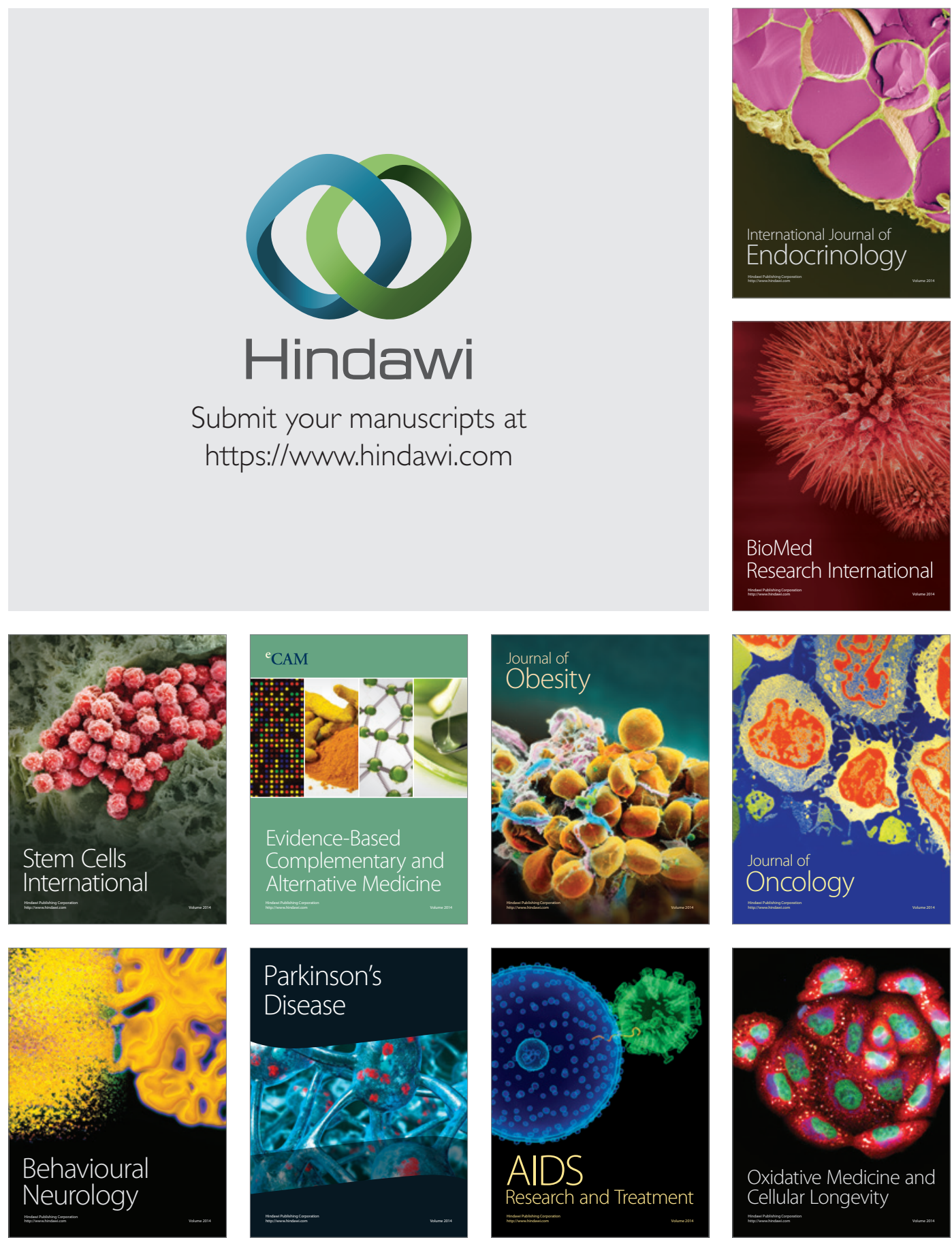